\title{
Efficient LCA based Keyword Search in XML Data
}

\author{
$\mathrm{Yu} \mathrm{Xu}$ \\ Teradata \\ San Diego, CA \\ yu.xu@teradata.com
}

\author{
Yannis Papakonstantinou \\ University of California, San Diego \\ San Diego, CA \\ yannis@cs.ucsd.edu
}

\begin{abstract}
Keyword search in XML documents based on the notion of lowest common ancestors $(L C A s)$ and modifications of it has recently gained research interest $[10,14,22]$. In this paper we propose an efficient algorithm called Indexed Stack to find answers to keyword queries based on XRank's semantics to LCA [10]. The complexity of the Indexed Stack algorithm is $O\left(k d\left|S_{1}\right| \log |S|\right)$ where $k$ is the number of keywords in the query, $d$ is the depth of the tree and $\left|S_{1}\right|(|S|)$ is the occurrence of the least (most) frequent keyword in the query. In comparison, the best worst case complexity of the core algorithms in [10] is $O(k d|S|)$. We analytically and experimentally evaluate the Indexed Stack algorithm and the two core algorithms in [10]. The results show that the Indexed Stack algorithm outperforms in terms of both $\mathrm{CPU}$ and I/O costs other algorithms by orders of magnitude when the query contains at least one low frequency keyword along with high frequency keywords. This is important in practice since the frequencies of keywords typically vary significantly.
\end{abstract}

\section{INTRODUCTION}

Keyword search in XML documents based on the notion of lowest common ancestors in the labeled trees modeled after the XML documents has recently gained research interest in the database community $[10,14,22,15,19]$. One important feature of keyword search is that it enables users to search information without having to know a complex query language or prior knowledge about the structure of the underlying data. Consider a keyword query $Q$ consisting of $k$ keywords $w_{1}, \ldots, w_{k}$. According to the LCA-based query semantics proposed in [10], named Exclusive Lowest Common Ancestors ( $E L C A$ ) in the sequel, the result of the keyword query $Q$ is the set of nodes that contain at least one occurrence of all of the query keywords either in their labels or in the labels of their descendant nodes, after excluding the occurrences of the keywords in the subtrees that already contain at least one occurrence of all the query keywords. For

Permission to make digital or hard copies of all or part of this work for personal or classroom use is granted without fee provided that copies are not made or distributed for profit or commercial advantage and that copies bear this notice and the full citation on the first page. To copy otherwise, to republish, to post on servers or to redistribute to lists, requires prior specific permission and/or a fee.

EDBT'08, March 25-30, 2008, Nantes, France.

Copyright 2008 ACM 978-1-59593-926-5/08/0003 ...\$5.00. example, the answers to the keyword query "XML David" on the data in Figure 1 is the node list $[0,0.2,0.2 .2,0.3,0.3 .2$, $0.3 .3,0.3 .4,0.4 .2]$. The answers show that "David" is an author of five papers that have "XML" in the titles (rooted at $0.2 .2,0.3 .2,0.3 .3,0.3 .4$ and 0.4 .2 ); and that "David" is the chair of two sessions that have "XML" in the titles (rooted at 0.2 and 0.3 ), and the chair of the conference (rooted at 0 ) whose name contains "XML". Notice that the node session with id 0.4 is not an ELCA answer since the only "XML" instance (node 0.4.2.1.1) under 0.4 is under one of its children (0.4.2) which already contains keyword instances of both "XML" and "David". Therefore under the exclusion requirement in the $E L C A$ definition, the session node 0.4 is not an ELCA answer. The node Conference rooted at 0 is an $E L C A$ answer since it contains the node 0.1.1 and the node 0.5.1 which are not under any child of the node 0 that contains instances of both keywords "XML" and "David".

We propose an efficient algorithm called Indexed Stack to answer keyword queries according to the ELCA query semantics proposed in XRank [10] with complexity of $O\left(k d\left|S_{1}\right| \log |S|\right)$ where $k$ is the number of keywords in the query, $d$ is the depth of the tree, $\left|S_{1}\right|(|S|)$ is the occurrence of the least (most) frequent keyword in the query. In comparison, the complexity of the core algorithms in [10] is $O(k d|S|)$ and $O\left(k^{2} d|S| p \log |S|+k^{2} d|S|^{2}\right)$ respectively where $p$ is the maximum number of children of any node in the tree. The algorithm in [10] with complexity $O\left(k^{2} d|S| p \log |S|+\right.$ $\left.k^{2} d|S|^{2}\right)$ is tuned to return only the top $m$ answers for certain queries where it may terminate faster than other algorithms. In particular, our contributions include:

- We propose an efficient algorithm, named Indexed Stack (IS) for keyword search in XML documents according to the ELCA semantics proposed in XRank [10]. Our analysis of the algorithm shows that the complexity of the proposed algorithm is $O\left(k d\left|S_{1}\right| \log |S|\right)$.

- Our experiments evaluate the Indexed Stack algorithm, and the algorithms in [10] and show that the Indexed Stack algorithm outperforms in terms of both CPU and I/O costs other algorithms by orders of magnitude when the query contains at least one low frequency keyword along with high frequency keywords.

In Section 2 we provide the ELCA query semantics and definitions used in the paper. Section 3 describes related work, with focus on LCA-based keyword search in XML documents based on the notation of lowest common ancestors $[10,14,22,15,19]$. Section 4 presents the Indexed Stack algorithm, and also provides the complexity analysis 


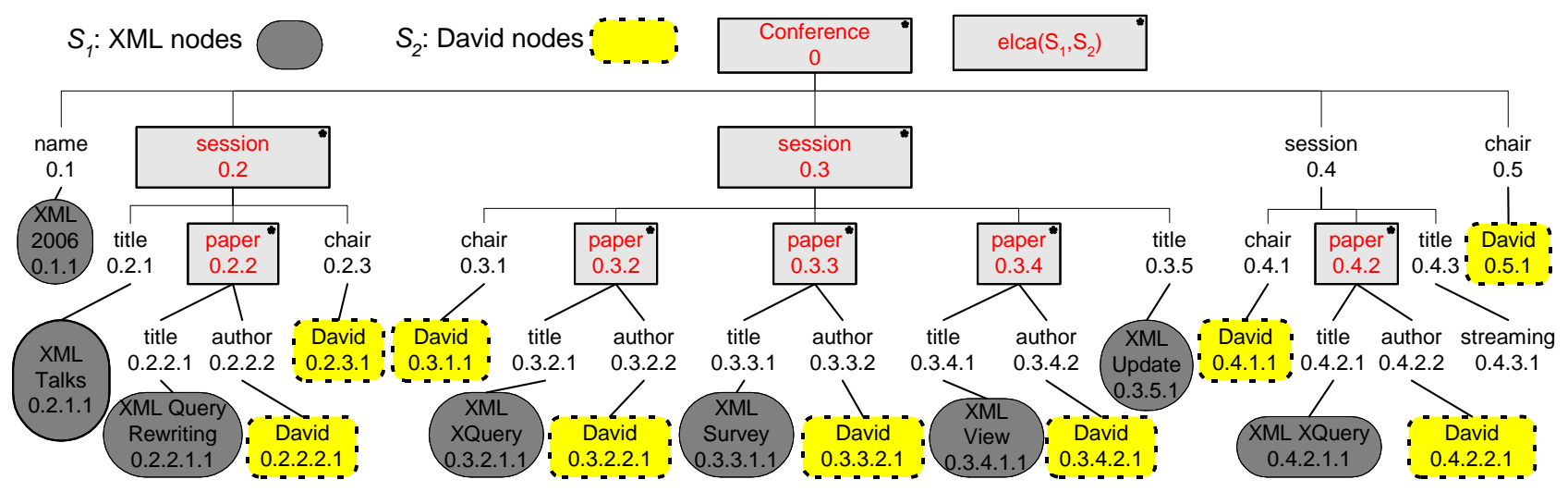

Figure 1: Example XML document

of the Indexed Stack algorithm and the algorithms in [10] for both main memory and disk accesses. Our experimental results comparing the Indexed Stack algorithm and the two core algorithms in [10] appear in Section 5. We conclude in Section 6 .

\section{ELCA QUERY SEMANTICS}

We model XML documents as trees using the conventional labeled ordered tree model. Each node $v$ of the tree corresponds to an XML element and is labeled with a tag $\lambda(v)$.

The notation $v \prec_{a} v^{\prime}$ denotes that node $v$ is an ancestor of node $v^{\prime} ; v \preceq_{a} v^{\prime}$ denotes that $v \prec_{a} v^{\prime}$ or $v=v^{\prime}$.

We first introduce the Lowest Common Ancestor ( $L C A)$ of $k$ nodes (sets) before we formally define the $E L C A$ query semantics.

The function $l c a\left(v_{1}, \ldots, v_{k}\right)$ computes the Lowest Common Ancestor $(L C A)$ of nodes $v_{1}, \ldots, v_{k}$. The $L C A$ of sets $S_{1}, \ldots, S_{k}$ is the set of $L C A$ 's for each combination of nodes in $S_{1}$ through $S_{k}$.

$$
l c a\left(S_{1}, \ldots, S_{k}\right)=\left\{l c a\left(n_{1}, \ldots, n_{k}\right) \mid n_{1} \in S_{1}, \ldots, n_{k} \in S_{k}\right\}
$$

For example, in Figure 1, lca $\left(S_{1}, S_{2}\right)=[0,0.2,0.2 .2,0.3$, $0.3 .2,0.3 .3,0.3 .4,0.4,0.4 .2]$.

A node $v$ is called an $L C A$ of sets $S_{1}, \ldots, S_{k}$ if $v \in l c a\left(S_{1}, \ldots, S_{k}\right)$.

A node $v$ is called an Exclusive Lowest Common Ancestor (ELCA) of $S_{1}, \ldots, S_{k}$ if and only if there exist nodes $n_{1} \in$ $S_{1}, \ldots, n_{k} \in S_{k}$ such that $v=l c a\left(n_{1}, \ldots, n_{k}\right)$ and for every $n_{i}(1 \leq i \leq k)$ the child of $v$ in the path from $v$ to $n_{i}$ is not an $L C \bar{A}$ of $S_{1}, \ldots, S_{k}$ itself nor ancestor of any $L C A$ of $S_{1}, \ldots, S_{k}$.

According to the ELCA query semantics proposed in XRank [10], the query result of a keyword query $Q$ consisting of $k$ keywords $w_{1}, \ldots, w_{k}$ is defined to be

$$
\operatorname{elca}\left(w_{1}, \ldots, w_{k}\right)=\operatorname{elca}\left(S_{1}, \ldots, S_{k}\right)
$$

where $\operatorname{elca}\left(S_{1}, \ldots, S_{k}\right)=\left\{v \mid \exists n_{1} \in S_{1}, \ldots, n_{k} \in S_{k}(v=\right.$ $l c a\left(n_{1}, \ldots, n_{k}\right) \wedge \quad \forall i(1 \leq i \leq k) \nexists x\left(x \in l c a\left(S_{1}, \ldots, S_{k}\right) \wedge\right.$ $\left.\left.\left.\operatorname{child}\left(v, n_{i}\right) \preceq_{a} x\right)\right)\right\}$ where $\bar{S}_{i}$ denotes the inverted list of $w_{i}$, i.e., the list of nodes sorted by id whose label directly contains $w_{i}$ and $\operatorname{child}\left(v, n_{i}\right)$ is the child of $v$ in the path from $v$ to $n_{i}$. The node $n_{i}$ is called an ELCA witness node of $v$ in $S_{i}$. Note that a node $v$ is an $E L C A$ of $S_{1}, \ldots, S_{k}$ if and only if $v \in \operatorname{elca}\left(S_{1}, \ldots, S_{k}\right)$.
Notice that the above definition is based on LCAs and is expressed differently than but it is equivalent to [10]. In Figure 1 elca ("XML", "David") $=\operatorname{elca}\left(S_{1}, S_{2}\right)=[0,0.2,0.2 .2$, $0.3,0.3 .2,0.3 .3,0.3 .4,0.4 .2]$. The node 0.1 .1 is an $E L C A$ witness node of the node 0 in $S_{1}$ and the node 0.5.1 is an ELCA witness node of the node 0 in $S_{2}$.

The Smallest Lowest Common Ancestor (SLCA) of $k$ sets $S_{1}, \ldots, S_{k}$ is defined to be

$$
\begin{aligned}
& \operatorname{slca}\left(S_{1}, \ldots, S_{k}\right)= \\
& \quad\left\{v \mid v \in l c a\left(S_{1}, \ldots, S_{k}\right) \wedge \forall v^{\prime} \in l c a\left(S_{1}, \ldots, S_{n}\right) \quad v \nprec v^{\prime}\right\}
\end{aligned}
$$

A node $v$ is called a Smallest Lowest Common Ancestor $(S L C A)$ of $S_{1}, \ldots, S_{k}$ if $v \in \operatorname{slca}\left(S_{1}, \ldots, S_{k}\right)$. Note that a node in $\operatorname{slca}\left(S_{1}, \ldots, S_{n}\right)$ cannot be an ancestor node of any other node in $\operatorname{slca}\left(S_{1}, \ldots, S_{n}\right)$.

In Figure 1, slca $\left(S_{1}, S_{2}\right)=[0.2 .2,0.3 .2,0.3 .3,0.3 .4,0.4 .2]$. Clearly $\operatorname{slca}\left(S_{1}, \ldots, S_{k}\right) \subseteq \operatorname{elca}\left(S_{1}, \ldots, S_{k}\right) \subseteq l c a\left(S_{1}, \ldots, S_{k}\right)$. For example, consider $S_{1}$ and $S_{2}$ in Figure 1 . The node 0.2 is not in slca $\left(S_{1}, S_{2}\right)$ but in elca $\left(S_{1}, S_{2}\right)$ and the node 0.4 is not in elca $\left(S_{1}, S_{2}\right)$ but in $\operatorname{lca}\left(S_{1}, S_{2}\right)$.

Similarly to [10, 22], each node is assigned a Dewey id $\operatorname{pre}(v)$ that is compatible with preorder numbering, in the sense that if a node $v_{1}$ precedes a node $v_{2}$ in the preorder left-to-right depth-first traversal of the tree then $\operatorname{pre}\left(v_{1}\right)<$ $\operatorname{pre}\left(v_{2}\right)$. Dewey numbers provide a straightforward solution to locating the $L C A$ of two nodes. The usual < relationship holds between any two Dewey numbers. Given two nodes $v_{1}$, $v_{2}$ and their Dewey numbers $p_{1}, p_{2}, l c a\left(v_{1}, v_{2}\right)$ is the node with the Dewey number that is the longest common prefix of $p_{1}$ and $p_{2}$. The cost of computing $l c a\left(v_{1}, v_{2}\right)$ is $O(d)$ where $d$ is the depth of the tree. For example, in Figure 1 $l c a(0.2 .2 .1 .1,0.2 .2 .2 .1)=0.2 .2$.

\section{RELATED WORK}

Extensive research has been done on keyword search in both relational and graph databases [9, 1, 11, 12, 3, 13]. There are works on keyword search on XML databases modeled as trees $[10,14,22,15,19,4,18]$. This work falls in this category. Finally $[16,6,7,18,20,21,14,2]$ integrate keyword search into XML query languages.

We focus on the following most closely related works: XRank ([10]), Schema-Free XQuery ([14]), XKSearch ([22]), XSeek ([15]), and Multiway SLCA-based keyword search ([19]), all of which base keyword search in XML on the no- 
tation of lowest common ancestors of the nodes containing keywords.

XRank ([10]) defines the answer to a keyword search query Q " $w_{1}, \ldots, w_{k}$ " to be elca $\left(S_{1}, \ldots, S_{k}\right)$ where $S_{i}$ is the inverted list of $w_{i}(1 \leq i \leq k)$. It also extends PageRank's ranking mechanism to XML by taking the nested structure of XML into account. Each node in the tree is assigned a precomputed ranking score which is independent of any keyword query. The ranking score of an answer node (i.e., an $E L C A$ node) $v$ to the query $Q$ is computed by XRank's aggregate ranking function which takes into account individual scores of the witness nodes of $v$ and the distance between the witness nodes and the answer nodes - the contribution of a witness node $x$ 's ranking to the node $v$ decays by the distance between $v$ and $x$. [10] proposes two core algorithms, DIL (Dewey Inverted List) and RDIL (Ranked Dewey Inverted List), to return the top $m$ answers from elca $\left(S_{1}, \ldots, S_{k}\right)$. Notice that the ranking functions and the search algorithms (DIL and RDIL) are independent of each other, in the sense that the same search algorithms could apply to other ranking functions ${ }^{1}$.

The DIL algorithm in [10] keeps an inverted list sorted by Dewey id for each keyword. DIL (conceptually) sort merges the $k$ inverted lists of the $k$ query keywords and reads each node $v$ in the sorted merged list in order. Intuitively it is easy to verify the correctness of the DIL algorithm since it reads all nodes in the $k$ inverted lists in document order and has enough information to determine whether a lowest common ancestor of $k$ nodes from the $k$ inverted list is an ELCA node or not. Notice that the DIL algorithm has to scan to the end of all inverted lists. The complexity of the DIL algorithm is $O(k d|S|)$ where $|S|$ is the size of the largest inverted list among $S_{1}, \ldots, S_{k}$ and $d$ is the depth of the tree.

The RDIL algorithm in [10] maintains two separate data structures: inverted lists sorted by the individual nodes' ranking score in descending order and $\mathrm{B}+$ trees built on inverted lists sorted by Dewey id in ascending order. The underlying assumption of RDIL is that higher ranked results ( $E L C A$ nodes) are likely to come from nodes in the front of inverted lists sorted by ranking score in descending order and query processing may terminate without scanning to the end of all of the inverted lists. RDIL works as follows:

1. it reads a node $v$ from the $k$ inverted lists sorted by rank, in round-robin fashion ${ }^{2}$.

2. then it uses the B+trees built on inverted lists sorted by Dewey id to find the lowest common ancestor $l$ that contains $v$ and all other keywords. The key observation is that given a node $v$, an inverted list $S$ sorted by document order and the $\mathrm{B}+$ tree $B T$ built on $S$, it takes only a single range scan ([8]) in $B T$ to find the node $v^{\prime}$ in $S$ whose id is the least that is greater than the id of $v$ such that either $v^{\prime}$ or its immediate predecessor in $S$ shares the longest common prefix with $v$ which is the Dewey id of $l$.

3. however the node $l$ produced in the second step may not be an $E L C A$ node. RDIL first determines whether each child of $l$ contains all keywords or not $(O(k d p \log |S|)$

\footnotetext{
${ }^{1}$ as long as the aggregate ranking functions are monotone with respect to individual keyword ranks (See Section 2.3 in [10] for more details).

${ }^{2}$ e.g., it reads a node from each inverted list in turn.
}

where $p$ is the maximum number of children of any node in the tree). Then for each keyword $w_{i}$, RDIL checks that keyword witness nodes of $l$ are not under any of its children that contain all keyword instances. The complexity of RDIL is $O\left(k^{2} d|S| p \log |S|+k^{2} d|S|^{2}\right)$.

Given a node $v$ in an inverted list, as can be seen from the above explanation, the RDIL algorithm does not completely scan other inverted lists in order to find an LCA node that contains $v$ and all other keywords. However, in order to guarantee correctness (not losing any answer nodes and not returning non-answer nodes), scan is repeatedly performed and that is why the complexity of the RDIL is high in the worst case. Furthermore, it is not guaranteed that individual nodes with higher ranking scores always lead to answer nodes with higher overall ranking score because the combination ranking function takes into account the distance between witness nodes and answer nodes. Moreover, given a keyword query, there is no practical way to determine a priori whether the DIL or the RDIL algorithm will perform better. The experiments in [10] have demonstrated that the performance of RDIL can be significantly worse than that of DIL for returning the top $m$ query answers. [10] proposes a hybrid algorithm which starts using RDIL and switches to DIL when it finds out that RDIL has spent too much time on answering the query.

XKSearch ([22]) defines the answers to a keyword query $Q$ of " $w_{1}, \ldots, w_{k}$ " to be $\operatorname{slca}\left(S_{1}, \ldots, S_{k}\right)$ where $S_{i}$ is the inverted list of the keyword $w_{i}$. The complexity of the Indexed Lookup Eager algorithm in [20] is $O\left(k d\left|S_{1}\right| \log |S|\right)$ and hence can be orders of magnitude better than the Stack based algorithm adopted from [10] or [14] when a query contains keywords of orders of magnitude of different frequencies. The key property of SLCA search in [22] is that, given two keywords $w_{1}$ and $w_{2}$ and a node $v$ that contains keyword $w_{1}$, one need not inspect the whole node list of keyword $w_{2}$ in order to discover potential solutions. Instead, one only needs to find the left and right match of $v$ in the list of $w_{2}$, where the left (right) match is the node with the greatest (least) id that is smaller (greater) than or equal to the id of $v$. The property generalizes to more than two keywords. [22] also extends the algorithm computing $\operatorname{slca}\left(S_{1}, \ldots, S_{k}\right)$ to compute all $L C A$ s of $k$ sets (i.e., lca $\left.\left(S_{1}, \ldots, S_{k}\right)\right)$. The intuition is that we can first compute all $S L C A$ nodes of $S_{1}, \ldots, S_{k}$. Then we visit every node $u$ in the path from every $S L C A$ node to the root and determine whether $u$ is a $L C A$ node or not. The complexity of the algorithm in [22] based on the above intuition to compute all $L C A \mathrm{~s}$ is $O\left(k d^{2}\left|S_{1}\right| \log |S|\right)$. We may attempt to compute elca $\left(S_{1}, \ldots, S_{k}\right)$ similarly. That is, in order to compute elca $\left(S_{1}, \ldots, S_{k}\right)$, we could do the following: (1) first compute slca $\left(S_{1}, \ldots, S_{k}\right)$ using the Indexed Lookup Eager algorithm in [22] whose complexity is $O\left(k d\left|S_{1}\right| \log |S|\right)$. (2) then for each $S L C A$ node $v$ computed in the first step, we walk up from $v$ to the root and determine whether each ancestor node $l$ of $v$ is an $E L C A$ node. However the difficulty is then that we have to perform the same expensive operations we described in the third step of the RDIL algorithm in [10] a few paragraphs before. Therefore the complexity of such an algorithm would be $O\left(k^{2} d|S| p \log |S|+k^{2} d|S|^{2}\right)$ where $p$ is the maximum number of children of any node in the tree.

Multiway SLCA-based keyword search in XML data [19] generalizes the SLCA query semantics to support keyword 
search beyond the AND semantics to include both AND and OR boolean operators. For AND-only SLCA query semantics, [19] notices that we may not need to completely scan the smallest keyword list for certain data instances. Instead certain keyword instances in the smallest keyword list can be skipped for faster processing, though the complexity of the algorithm proposed in [19] is still $O\left(k d\left|S_{1}\right| \log |S|\right)$.

Schema-Free XQuery ([14]) uses the idea of Meaningful $L C A$ (MLCA), similar to $S L C A$, and proposes a stack based sort merge algorithm which scans to the end of all inverted lists. The complexity of the algorithm in [14] is the same as that of DIL $(O(k d|S|))$. [14] shows that keyword search functionality can be easily integrated into the structured query language XQuery as built-in functions, enabling users to query XML documents based on partial knowledge they may have over underlying data with different and potentially evolving structures. The recall and precision experiments in [14] shows that it is possible to express a wide variety of queries in a schema-free manner and have them return correct results over a broad diversity of schema. The demonstrated integration of $M L C A$ based keyword search functionality into XQuery can also apply to the $E L C A$ query semantics.

XSeek [15] studies the problem of inferring the most relevant return nodes without elicitation of user preferences. It works for data with or without schema information. XSeek generates two types of nodes: return nodes that can be inferred explicitly by analyzing keyword match patterns; and return nodes that can be inferred implicitly by considering both keyword match patterns and XML data structure. The experiments show that XSeek generates results with improved precision and recall over prior approaches with reasonable cost.

In this paper we will only focus on the algorithmic aspects of the problem of efficiently finding answers to keyword queries in XML documents. we will not attempt a comparison of the quality of different query semantics.

Intuitively answering a keyword query according to the $E L C A$ query semantics is more computationally challenging than according to the $S L C A$ query semantics. In the latter the moment we know a node $l$ has a child $c$ which contains all keywords, we can immediately determine that the node $l$ is not a $S L C A$ node. However we cannot determine that $l$ is not an $E L C A$ node because $l$ may contain keyword instances that are not under $c$ and are not under any node that contains all keywords. Notice that given the same query, the size of the answers of the $S L C A$ semantics cannot be more than that of the ELCA semantics because $\operatorname{slca}\left(S_{1}, \ldots, S_{k}\right) \subseteq \operatorname{elca}\left(S_{1}, \ldots, S_{k}\right)$.

In this paper, we propose an efficient algorithm, Indexed Stack algorithm (IS), which takes advantage of the benefits of both stack based algorithms and indexed lookup based algorithms. The complexity is $O\left(k d\left|S_{1}\right| \log |S|\right)$.

\section{INDEXED STACK ALGORITHM (IS)}

This section presents the Indexed Stack (IS) algorithm that computes elca $\left(S_{1}, \ldots, S_{k}\right)$. We choose $S_{1}$ to be the smallest among $S_{1}, \ldots, S_{k}$ since elca $\left(S_{1}, \ldots, S_{k}\right)$

$=\operatorname{elca}\left(S_{j_{1}}, \ldots, S_{j_{k}}\right)$, where $j_{1}, . ., j_{k}$ is any permutation of $1,2, \ldots, k$, and there is a benefit in using the smallest list as $S_{1}$ as we will see in the complexity analysis of the algorithm. We assume $|S|$ denotes the size of the largest inverted list. The Indexed Stack algorithm, leveraging key tree properties described in this section, starts from the smallest list $S_{1}$, visits each node in $S_{1}$, but does not need to access every node in other lists. It achieves high efficiency, especially when the smallest list is significantly smaller than the largest list.

The algorithm's efficiency is based on first discovering the nodes of a set elca_can $\left(S_{1} ; S_{2}, \ldots, S_{k}\right)$ (short for $E L C A$ Candidates) defined in Section 4.1, which is a superset of elca $\left(S_{1}, \ldots, S_{k}\right)$ but can be computed efficiently in $O\left(k d\left|S_{1}\right| \log |S|\right)$, as shown in Section 4.2. Section 4.3 describes an efficient function isELCA() that determines whether a given node of elca_can $\left(S_{1} ; S_{2}, \ldots, S_{k}\right)$ is a member of elca $\left(S_{1}, \ldots, S_{k}\right)$. Section 4.4 presents a stack-based algorithm that puts together the computation of elcan_can and isELCA, avoiding redundant computations. Section 4.4 also presents the complexity analysis of the algorithm.

\subsection{The ELCA candidate set elca_can()}

We define next the set elca_can $\left(S_{1} ; S_{2}, \ldots, S_{k}\right)$, whose members are called ELCA_CAN nodes (of $S_{1}$ among $S_{2}$, $\left.\ldots, S_{k}\right)$.

$$
\text { elca_can }\left(S_{1} ; S_{2}, \ldots, S_{k}\right)=\bigcup_{v_{1} \in S_{1}} \operatorname{slca}\left(\left\{v_{1}\right\}, S_{2}, \ldots, S_{k}\right)
$$

Note that a node $v$ is an $E L C A \_C A N$ node iff there exist $n_{1} \in S_{1}, \ldots, n_{k} \in S_{k}$ such that $v=l c a\left(n_{1}, \ldots, n_{k}\right)$ and there must not exist $n_{2}^{\prime} \in S_{2}, \ldots, n_{k}^{\prime} \in S_{k}$ such that $v^{\prime}=$ $l c a\left(n_{1}, n_{2}^{\prime}, \ldots, n_{k}^{\prime}\right)$ and $v \prec_{a} v^{\prime}$. Every $n_{i}(1 \leq i \leq k)$ is called an ELCA_CAN witness node of $v$ in $S_{i}$.

For example, in Figure 1 elca_can $\left(S_{1} ; S_{2}\right)=[0,0.2,0.2 .2$, $0.3,0.3 .2,0.3 .3,0.3 .4,0.4 .2]$. Next, consider the $E L C A \_C A N$ node 0.2. The nodes 0.2 .1 .1 and 0.2 .2 .2 .1 are its witness nodes in $S_{1}$ and $S_{2}$ respectively. However the node 0.2.2.1.1 is not a witness node for 0.2 in $S_{1}$. This is because although the node 0.2 is the $L C A$ of the node 0.2.2.1.1 from $S_{1}$ and the node 0.2.3.1 from $S_{2}$, there exists the node 0.2.2.2.1 from $S_{2}$ such that the $L C A$ of 0.2 .2 .1 .1 and 0.2.2.2.1 (i.e., 0.2.2) is a descendant of 0.2 .

Note that elca_can $\left(S_{1} ; S_{2}, \ldots, S_{k}\right)$ may contain nodes that are ancestors of other nodes of elca_can $\left(S_{1} ; S_{2}, \ldots, S_{k}\right)$. The following inclusion relationship between elca and elca_can applies.

\section{PROPERTy 1.}

$\begin{aligned} \forall i \in & {[1, \ldots, k], } \\ \quad & \quad l c a\left(S_{1}, \ldots, S_{k}\right) \subseteq \text { elca_can }\left(S_{i} ; S_{1}, \ldots, S_{i-1}, S_{i+1}, \ldots, S_{k}\right) .\end{aligned}$

Proof. If $v \in \operatorname{elca}\left(S_{1}, \ldots, S_{k}\right)$, there must exist $E L C A$ witness nodes $n_{1} \in S_{1}, \ldots, n_{k} \in S_{k}$ such that $v=l c a\left(n_{1}, \ldots, n_{k}\right)$ and there must not exist $n_{1}^{\prime} \in S_{1}, \ldots, n_{i-1}^{\prime} \in S_{i-1}, n_{i+1}^{\prime} \in$ $S_{i+1}, \ldots, n_{k}^{\prime} \in S_{k}$ such that $v^{\prime}=l c a\left(n_{1}^{\prime}, \ldots, n_{i-1}^{\prime}, n_{i}, n_{i+1}^{\prime}, \ldots, n_{k}^{\prime}\right)$ and $v \prec_{a} v^{\prime}$ (Otherwise $n_{i}$ cannot be an ELCA witness node of $v$ ). Thus $v \in$ elca_can $\left(S_{i} ; S_{1}, \ldots, S_{i-1}, S_{i+1}, \ldots, S_{k}\right)$ by definition.

Of particular importance is the instantiation of the above property for $i=1$ (i.e., elca $\left(S_{1}, \ldots, S_{k}\right) \subseteq$ elca_can $\left.\left(S_{1} ; S_{2}, \ldots, S_{k}\right)\right)$ since elca_can $\left(S_{1} ; S_{2}, \ldots, S_{k}\right)$ has the most efficient computation (recall $S_{1}$ is the shortest inverted list).

In Figure 1, elca $\left(S_{1}, S_{2}\right)$ and elca_can $\left(S_{1} ; S_{2}\right)$ happen to be the same. However if we remove the node 0.3.1.1 from the tree in Figure 1, then elca_can $\left(S_{1} ; S_{2}\right)$ stays the same but the node 0.3 would not be in elca $\left(S_{1}, S_{2}\right)$ anymore. Therefore, it would be elca $\left(S_{1}, S_{2}\right) \subset$ elca_can $\left(S_{1} ; S_{2}\right)$. 
For presentation brevity, we define elca_can $(v)$ for $v \in S_{1}$ to be the node $l$ where $\{l\}=$ elca_can $\left(\{v\} ; S_{2}, \ldots, S_{k}\right)=$ $\operatorname{slca}\left(\{v\}, S_{2}, \ldots, S_{k}\right)$. The node elca_can $(v)$ is called the exclusive lowest common ancestor candidate or ELCA_CAN of $v$ (in sets of $S_{2}, \ldots, S_{k}$ ). Note that each node in $l c a\left(\{v\}, S_{2}, \ldots, S_{k}\right)$ is either an ancestor node of $v$ or $v$ itself and $e l c a \_c a n(v)$ is the lowest among all nodes in lca $\left(\{v\}, S_{2}, \ldots, S_{k}\right)$. For instance, consider $S_{1}$ and $S_{2}$ in Figure 1. elca_can $(0.1 .1)=0$, elca_can $(0.2 .1 .1)=0.2$, elca_can $(0.2 .2 .1 .1)=0.2 .2$, elca_can $(0.3 .2 .1 .1)=0.3 .2$, elca_can $(0.3 .3 .1 .1)=0.3 .3$, elca_can $(0.3 .4 .1 .1)=0.3 .4$, elca_can $(0.3 .5 .1)=0.3$ and elca_can $(0.4 .2 .1 .1)=0.4 .2$.

\subsection{Computing elca_can(v)}

In this section we describe how prior work $([22])$ can be extended to efficiently compute elca_can $(v)$ in the interest of completeness and clarity.

Let us assume that we want to compute $\operatorname{slca}\left(\{v\}, S_{2}\right)$ where $S_{2}=\left\{u_{1}, \ldots, u_{n}\right\}$. The key observation in [22] is that the witness node in $S_{2}$ for $\operatorname{slca}\left(v_{1}, S_{2}\right)$ must be one of the two closest nodes (in document order) to $v$ among all nodes in the set $S_{2}$. We can efficiently find the only two nodes of $\left\{u_{1}, \ldots, u_{n}\right\}$ that are candidates for witnessing the $S L C A$, by using two important functions: the function $r m(v, S)$ computes the right match of $v$ in a set $S$, that is the node of $S$ that has the smallest id that is greater than or equal to $\operatorname{pre}(v) ; \operatorname{lm}(v, S)$ computes the left match of $v$ in a set $S$, that is the node of $S$ that has the biggest id that is less than or equal to $\operatorname{pre}(v)$. The function $\operatorname{rm}(v, S)$ $(\operatorname{lm}(v, S))$ returns null when there is no right (left) match node. For example, consider again $S_{1}$ and $S_{2}$ in Figure 1 and the node $v=0.3 .2 .1 .1$ from $S_{1}$. The right match for $v$ in $S_{2}$ is the node 0.3.2.2.1, and the left match for $v$ in $S_{2}$ is the node 0.3.1.1. Consequently $\operatorname{slca}\left(\{v\}, S_{2}\right)$ is the lower node from $l c a\left(v, r m\left(v, S_{2}\right)\right)$ and $l c a\left(v, \operatorname{lm}\left(v, S_{2}\right)\right)$. Consider again $S_{1}, S_{2}$, and $v=0.3 .2 .1 .1$ from $S_{1}$ in Figure 1, elca_can $(0.3 .2 .1 .1)=0.3 .2$. This is because

$l c a\left(v, r m\left(v, S_{2}\right)\right)=l c a(v, 0.3 .2 .2 .1)=0.3 .2$,

$l c a\left(v, \operatorname{lm}\left(v, S_{2}\right)\right)=l c a(v, 0.3 .1 .1)=0.3$, and $0.3 \prec{ }_{a} 0.3 .2$.

The cost of computing $r m(v, S)(\operatorname{lm}(v, S))$ is $O(d \log |S|)$ since it takes $O(\log |S|)$ steps (each step being a Dewey number comparison) to find the right (left) match node and the cost of comparing the Dewey ids of two nodes is $O(d)$.

The key point in [22] applies to the computation of $\operatorname{slca}\left(\{v\}, S_{2}, \ldots, S_{k}\right)$. The node $e l c a \_c a n(v)$ (i.e., slca $\left.\left(\{v\} ; S_{2}, \ldots, S_{k}\right)\right)$ can be efficiently computed as follows: First we compute the (unique) $S L C A v_{2}$ of $v$ and of the nodes of $S_{2}$. It continues by iteratively computing the (unique) $S L C A v_{i}$ of $v_{i-1}$ and $S_{i}$, until $i$ becomes $k$. The node $v_{k}$ is the result.

Notice though that the nodes of elca_can $\left(S_{1} ; S_{2}, \ldots, S_{k}\right)$ may be obtained out of order by applying the above computation on each node in $S_{1}$. For example in Figure 1, elca_can $(0.3 .2 .1 .1)=0.3 .2$ and elca_can $(0.3 .5 .1)=0.3$. Thus the $E L C A \_C A N$ node 0.3 is computed after the $E L C A \_C A N$ node 0.3.2. The time complexity of computing elca_can $(v)$ is $O(k d \log |S|)$.

\subsection{Determine whether an $E L C A \_C A N$ node is an $E L C A$ node}

This section presents the function isELCA which is used to determine whether an ELCA_CAN node $v$ is an ELCA node or not. Let child_elcacan $(v)$ be the set of children of

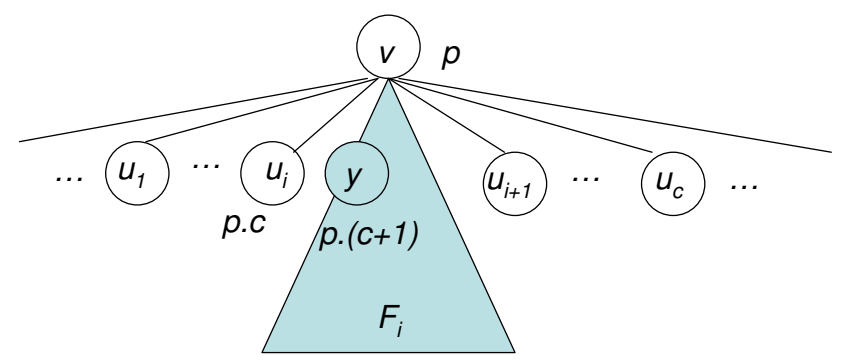

Figure 2: $v$ and its $E L C A \_C A N$ children

$v$ that contain all keyword instances. Equivalently child_elcacan $(v)$ is the set of child nodes $u$ of $v$ such that either $u$ or one of $u$ 's descendant nodes is an ELCA_CAN node, i.e.,

$$
\begin{aligned}
& \operatorname{child\_ elcacan}(v)=\{u \mid u \in \operatorname{child}(v) \wedge \\
& \left.\quad \exists x \quad\left(u \preceq_{a} x \wedge x \in \text { elca_can }\left(S_{1} ; S_{2}, \ldots, S_{k}\right)\right)\right\}
\end{aligned}
$$

where $\operatorname{child}(v)$ is the set of child nodes of $v$. We use $E L C A \_C A N$ in the above definition of child_elcacan $(v)$ because we can efficiently compute elca_can $\left(S_{1} ; S_{2}, \ldots, S_{k}\right)$ as discussed in Section 4.2. For $S_{1}$ and $S_{2}$ of the running example in Figure 1, child_elcacan $(0)=[0.2,0.3,0.4]$ and child_elcacan $(0.2)=[0.2 .2]$.

Assume child_elcacan $(v)$ is $\left\{u_{1}, \ldots, u_{c}\right\}$ (See Figure 2). By definition, an ELCA node $v$ must have ELCA witness nodes $n_{1}, \ldots, n_{k}$ such that $n_{1} \in S_{1}, \ldots, n_{k} \in S_{k}$ and every $n_{i}$ is not in the subtrees rooted at the nodes from child_elcacan $(v)$.

To determine whether $v$ is an $E L C A$ node, we probe every $S_{i}$ to see if there is a node $x_{i} \in S_{i}$ such that $x_{i}$ is either in the forest under $v$ to the left of the path $v u_{1}$, or in the forest under $v$ to the right of the path $v u_{c}$, or in any forest $F_{i}$ that is under $v$ and between the paths $v u_{i}$ and $v u_{i+1}$, $i=1, \ldots, c-1$. The last case can be checked efficiently by finding the right match $r m\left(y, S_{i}\right)$ of the node $y$ in $S_{i}$ where $y$ is the immediate right sibling of $u_{i}$ among the children of $v$. Assume pre $(v)=p$, pre $\left(u_{i}\right)=p . c$ where $c$ is a single number, then pre $(y)=p \cdot(c+1)$, as shown in Figure 2. Let the right match of $y$ in $S_{i}$ be $x$ (i.e., $x=r m\left(y, S_{i}\right)$ ). Then $x$ is a witness node in the forest $F_{i}$ if and only if $\operatorname{pre}(x)<\operatorname{pre}\left(u_{i+1}\right)$.

Given the list $c h$ which is the list of nodes in child_elcacan $(v)$ sorted by id, the function isELCA $(v, c h)$ (Figure 3) returns true if $v$ is an $E L C A$ node by applying the operations described in the previous paragraph. As an example, consider the query "XML David" and the inverted lists $S_{1}$ and $S_{2}$ in Figure 1. child_elcacan $(0)=[0.2,0.3,0.4]$. We will see how is $\operatorname{ELCA}(0,[0.2,0.3,0.4])$ works and returns true. In this example, the number of keywords is two $(k=2)$ and $|c h|=3$. First the function isELCA searches and finds the existence of an $E L C A$ witness node (i.e., the node 0.1.1) for 0.2 in $S_{1}$ in the subtree rooted under 0 to the left of the path from 0 to 0.2 (0.2 is the first child ELCA_CAN node of 0$)$. Then the function searches the existences of an $E L C A$ witness node in $S_{2}$ for 0 in the forest to the left of the path from 0 to 0.2 ; in the forest between the path from 0 to 0.2 and the path from 0 to 0.3 ; in the forest between the path from 0 to 0.3 and the path from 0 to 0.4 ; in the forest to the right of the path from 0 to 0.4. All of the above searches fail except that 


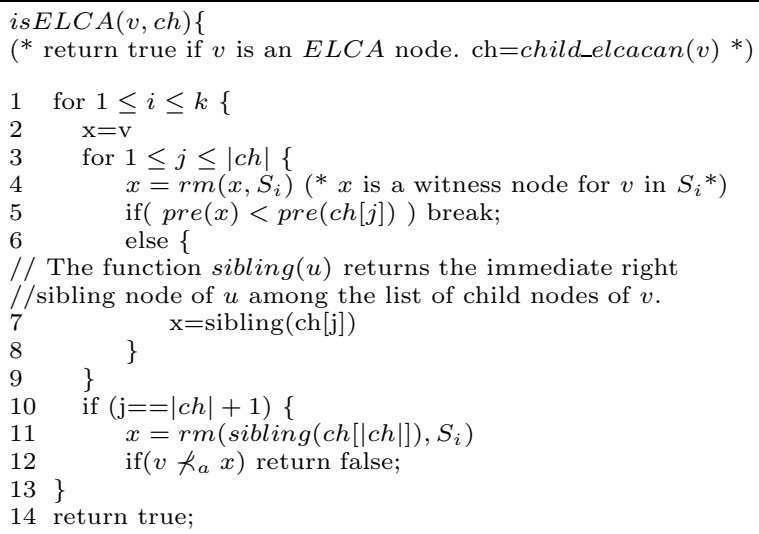

Figure 3: Determine whether an ELCA_CAN node is an $E L C A$ node

the last search successfully finds a witness node (0.5.1) for 0.2 in $S_{2}$. Therefore, isELCA(0, [0.2, 0.3, 0.4]) returns true.

The time complexity of $i s E L C A(v, c h)$ is $O(k d \log |S| \mid$ child_elcacan $(v) \mid)$ (line 1, 3 and 4$)$.

\subsection{Indexed Stack Algorithm}

In Section 4.1 we stated that elca_can $\left(S_{1} ; S_{2}, \ldots, S_{k}\right)$ is a superset of elca $\left(S_{1}, \ldots, S_{k}\right)$. Section 4.2 described how to efficiently compute elca_can $\left(S_{1} ; S_{2}, \ldots, S_{k}\right)$ and Section 4.3 described how to efficiently check whether an $E L C A \_C A N$ node in elca_can $\left(S_{1} ; S_{2}, \ldots, S_{k}\right)$ is an $E L C A$ node, when the list of child nodes of $v$ that contain all keyword instances are given. Therefore, the only missing part of efficient computation of elca $\left(S_{1}, \ldots, S_{k}\right)$ is how to compute child_elcacan $(v)$ for each $E L C A \_C A N$ node $v$. Since we can easily compute child_elcacan $(v)$ if we know every $E L C A \_C A N$ node $x_{i}$ under $v^{3}$, we can just compute all ELCA_CAN nodes and then compute child_elcacan $(v)$ for each $E L C A \_C A N$ node $v$.

A straightforward approach would compute all ELCA_CAN nodes and store them in a tree which keeps only the original ancestor-descendant relationships of all ELCA_CAN nodes in the input document. As an example, such a tree describing all ELCA_CAN nodes in Figure 1 is shown in Figure 4. Note that though 0.4 .2 is a descendant of 0 in Figure 1, it is a child of 0 in Figure 4.

A straightforward algorithm to compute elca $\left(S_{1}, \ldots, S_{k}\right)$ works as follows where $T S$ is a tree structure initialized to empty:

1. For each node $v$ in $S_{1}$, compute $l=e l c a \_c a n(v)$ based on Section 4.2 and do TS.insert $(l)$ which inserts $l$ to the appropriate place in $T S$ based on l's ancestordescendant relationship with nodes already inserted in $T S$. The tree in Figure 4 shows the result from this step for computing elca $\left(S_{1}, S_{2}\right)$ in Figure 1.

2. For each node $l$ in $T S$ check whether $l$ is an $E L C A \_C A N$ node or not by calling isELCA(l,child_elcacan $(l))$ where child_elcacan $(l)$ can be easily computed from the list of child nodes of $l$ in $T S$.

\footnotetext{
${ }^{3}$ child_elcacan $(v)$ is the set of child nodes $u_{i}$ of $v$ on the paths from $v$ to $x_{i}$, which can be efficiently computed with Dewey numbers.
}

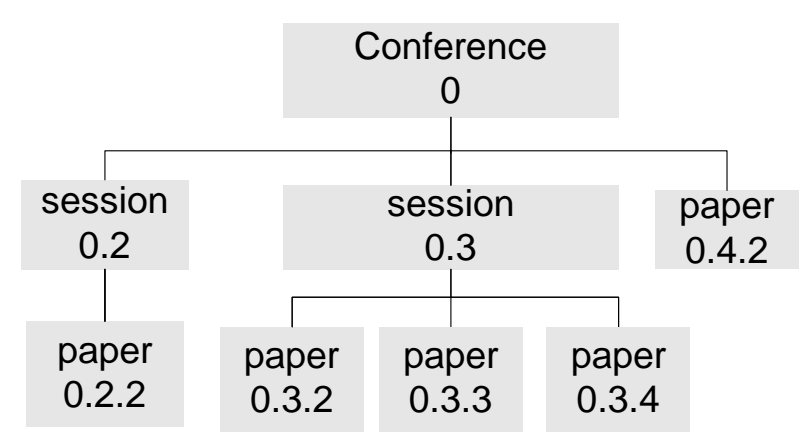

Figure 4: The tree structure of all $E L C A \_C A N$ nodes in Figure 1

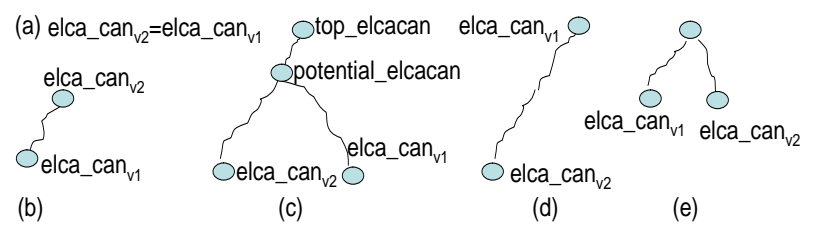

Figure 5: Relationships between any two nodes $l$ and $s$

However the above approach has the following disadvantages:

- the complexity of the approach is $O\left(d\left|S_{1}\right|^{2}+\left|S_{1}\right| k d \log |S|\right)$ where the $O\left(d\left|S_{1}\right|^{2}\right)$ component comes from the cost of creating and maintaining the tree structure;

- and all $\left(O\left(\left|S_{1}\right|\right)\right)$ ELCA_CAN nodes have to be computed first and kept in memory before we can start to recognize any $E L C A$ nodes.

Instead, a "one pass" stack-based algorithm, whose complexity is $O\left(\left|S_{1}\right| k d \log |S|\right)$, is presented in Figure 6. The Indexed Stack algorithm does not have to keep all $E L C A \_C A N$ nodes in memory; it uses a stack whose depth is bounded by the depth of the tree based on some key tree properties. At any time during the computation any node in the stack is a child or descendant node of the node below it (if present) in the stack. Therefore the nodes from the top to the bottom of the stack at any time are from a single path in the input tree.

We will first present the Indexed Stack algorithm, illustrated with a running example, then discuss at the end of this section optimization techniques in the implementation of the algorithm.

\subsubsection{Algorithm Description}

We go through every node $v_{1}$ in $S_{1}$ in order, compute elca_can $n_{v_{1}}=$ elca_can $\left(v_{1}\right)$ and create a stack entry stackEntry consisting of elca_can $n_{v_{1}}$. If the stack is empty, we simply push stackEntry to the stack to determine later whether elca_can $v_{v_{1}}$ is an ELCA node or not. If the stack is not empty, what the algorithm does depends on the relationship between stackEntry and the top entry in the stack. The algorithm either discards stackEntry or pushes stackEntry to the stack (with or without first popping out some stack entries). The algorithm does not need to look at any other non top entry in the stack at any time and only determines 
whether an ELCA_CAN node is an ELCA node at the time when a stack entry is popped out.

Each stack entry stackEntry created for a node $v_{1}$ in $S_{1}$ has the following three components.

- stackEntry.elca_can is elca_can $\left(v_{1}\right)$;

- stackEntry.CH records the list of child or descendant ELCA_CAN nodes of stackEntry.elca_can seen so far, which will be used by isELCA() to determine whether stackEntry.elca_can is an ELCA node at the time when this entry is popped out from the stack;

- and stackEntry.SIB (short for siblings) is the list of ELCA_CAN nodes before stackEntry.elca_can (in document order) such that the $L C A$ node of nodes from the list and stackEntry.elca_can potentially can be an $E L C A \_C A N$ node that has not been seen so far.

Let us illustrate the need for and role of stackEntry.SIB with the running example "XML David". Before we compute elca_can $(0.3 .5 .1)=0.3$, we have already computed 0.3.2, 0.3.3, 0.3.4 as ELCA_CAN nodes which are the child $E L C A \_C A N$ nodes of 0.3 . We have to store these three ELCA_CAN nodes in order to determine whether 0.3 is an $E L C A$ node or not before we see 0.3 in the processing, which is achieved by first storing 0.3 .2 in the $S I B$ component of the stack entry associated with 0.3 .3 and then storing 0.3 .2 and 0.3 .3 in the $S I B$ component of the stack entry associated with 0.3.4 (after the stack entry associated with 0.3 .3 is popped out) during the processing before we see 0.3 . Note that if the node 0.3.1.1 was not in the tree in Figure 1, we would still see 0.3 in the processing as an ELCA_CAN node and still see 0.3 after $0.3 .2,0.3 .3$, and 0.3 .4 , but then 0.3 would not be an ELCA node, which could be determined only if we have kept the information that $0.3 .2,0.3 .3$ and 0.3.4 are ELCA_CAN nodes until we see 0.3 and know that 0.3 would not have any child or descendant ELCA_CAN nodes in the processing later after we see 0.3 . It is possible that we would not see 0.3 at all in the processing (i.e., if the node 0.3.5.1 was not in the tree, 0.3 would be not be an ELCA_CAN node) in which case we still need to keep 0.3.2, 0.3.3 and 0.3.4 until the point we are sure that those nodes cannot be child or descendant of any other ELCA_CAN nodes.

Figure 6, which presents the Indexed Stack pseudo-code, and Figure 7, which has snapshots of the stack during operation of the algorithm, also include an entry

stackEntry.witNodes, which we temporarily ignore, as it is used only in the optimization version of the algorithm, described at the end of this section.

For each node $v_{1}$ in $S_{1}$ (line 2), the Indexed Stack algorithm computes elca_can $v_{1}=e l c a \_c a n\left(v_{1}\right)$ as discussed in Section 4.2 (line 4). We create a stack entry stackEntry consisting of elca_can $v_{1}$ (line 6). If the stack is empty (line 7), we simply push stackEntry to the Stack to determine later whether elca_can $v_{1}$ is an ELCA node or not. If the stack is not empty, let the node at the top of the stack be elca_can $v_{v_{2}}$ (line 9-10). Figure 5 shows the only five relationships the two ELCA_CAN nodes elca_can $v_{v_{2}}$ and elca_can $v_{v_{1}}$ (in fact any two nodes) can have.

- In the first case where elca_can $v_{v_{1}}$ and elca_can $v_{v_{2}}$ are the same (Figure 5(a), line 11), elca_can $v_{1}$ is discarded.
- In the second case where elca_can $v_{2}$ is an ancestor of elca_can $v_{v_{1}}$ (Figure 5(b)), we push stackEntry to the stack to determine later whether elca_can $v_{1}$ is an ELCA node or not (line 12).

- In the third case (Figure 5(c)) where elca_can $n_{v_{2}}$ and elca_can $v_{1}$ have no ancestor-descendant relationship and $e l c a \_c a n_{v_{1}}$ appears after elca_can $v_{v_{2}}$ in document order (line 13), we pop the top stack entry repeatedly (line 14) until either the stack is empty or the $E L C A \_C A N$ node of the top entry in the Stack (named top_elcacan in Figure 5(c), line 15) is an ancestor of elca_can $_{v_{1}}$ by calling the function popStack(). When a stack entry is popped out, the $E L C A_{-} C A N$ node in the stack entry is checked whether it is an $E L C A$ node or not (by isELCA()). Note that there will not be any $E L C A \_C A N$ node in later processing that can be a child or descendant node of any popped out $E L C A \_C A N$ node. That is why we can pop out those entries and check for ELCA nodes. Let popEntry be the last popped out entry and potential_elcacan be the LCA of popEntry.elca_can and elca_can $v_{v_{1}}$ (Figure 5(c), line 16). If the stack is not empty and the top stack entry's node top_elcacan is an ancestor of potential_elcacan (Figure 5(c), line 17), then we set the $S I B$ list associated with elca_can $v_{1}$ to be the concatenation of the $S I B$ list in popEntry and popEntry.elca_can (line 18). We then push stackEntry to the stack (line 19). The reason that we need to carry on the nodes stored in the $S I B$ component of popEntry to stackEntry was explained a few paragraphs before in the example illustrating the need for and role of the $S I B$ component in a stack entry. During the processing of the example, at one point elca_can $v_{2}$ is 0.3.2, elca_can $v_{1}$ is 0.3 .3 , potential_elcacan is 0.3 , top_elcacan is 0 , and after the stack entry for 0.3 .2 is popped out, 0.3 .2 is stored in the $S I B$ component of the stack entry for 0.3.3. Notice that potential_elcacan could be a node that we have not seen so far in the processing (i.e., it has not been computed as an $E L C A \_C A N$ node) and it could be an ELCA_CAN and an $E L C A$ node. Although we have guessed its existence here, it may or may not appear later in the processing. That is why we need to carry elca_can $n_{v_{2}}$ and nodes in the $S I B$ component of elca_can $v_{v_{2}}$ to the $S I B$ component of elca_can $v_{v_{1}}$ for potential_elcacan.

- In the fourth case where elca_can $v_{1} \prec_{a}$ elca_can $n_{v_{2}}$ (line 21, Figure 5(d)), it is certain that elca_can $v_{v_{2}}$ has no more descendant ELCA_CAN nodes. Thus we pop from the stack repeatedly until either the stack is empty or the ELCA_CAN node in the top entry is an ancestor of elca_can $v_{1}$ (line 22). Again, the ELCA_CAN node in each popped out entry is checked whether it is an $E L C A$ node or not. Let the last popped out entry be popEntry (line 22). We copy the SIB list in popEntry and popEntry.elca_can to the $\mathrm{CH}$ field of elca_can $n_{v_{1}}$ (line 23). Then stackEntry is pushed to the top of the stack (line 24). Notice that nodes stored in the $S I B$ field by the processing in the third case are used in the fourth case to set the $\mathrm{CH}$ field.

- The fifth case, where elca_can $v_{1}$ and elca_can $v_{v_{2}}$ have no ancestor-descendant relationship and elca_can $v_{1}$ ap- 


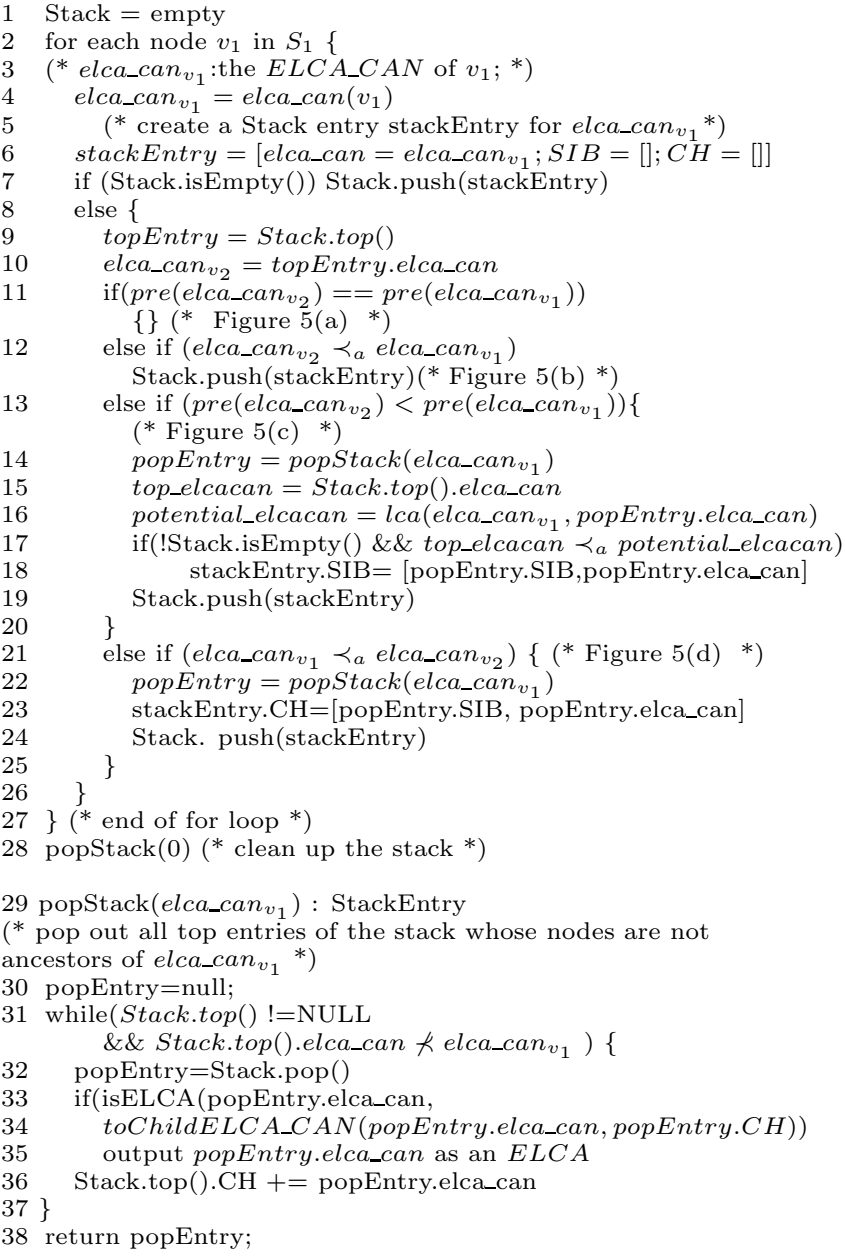

Figure 6: The Indexed Stack Algorithm

pears before elca_can $v_{2}$, is not possible in the computation when $S_{1}$ is sorted in document order.

Now we discuss the details of the function popStack $\left(e l c a_{-}{ }_{c a n} n_{v_{1}}\right)$ (called in the processing of the third and fourth cases in Figure 5). It repeatedly pops out the top entry (line 31) until the ELCA_CAN node in the top entry is an ancestor of elca_can $v_{1}$ or the stack becomes empty. Each popped out entry is checked on whether it contains an $E L C A$ node or not by calling the function isELCA presented in Section 4.3 (line 33). Notice that the function toChildELCA_CAN $(v, L)$ inputs a node $v$ and a list $L$ each node of which is a child or descendant $E L C A \_C A N$ node of $v$ and returns child_elcacan $(v)$. Each popped out node is added to the top entry's $C H$ field (line 36) because at any time any $E L C A \_C A N$ node in a stack entry is a child or descendant node of the $E L C A \_C A N$ node in the stack entry below it (if present).

The time complexity of the Indexed Stack algorithm is $O\left(\left|S_{1}\right| k d \log |S|\right)$ where $k$ is the number of keywords in the query, $d$ is the depth of the tree and $\left|S_{1}\right|(|S|)$ is the occurrence of the least (most) frequent keyword in the query. The time complexity comes from two primitive operations: elca_can() and isELCA(). The total cost of calling elca_can $(v)$ is $O\left(k d\left|S_{1}\right| \log |S|\right)$ as discussed in Section 4.2. The cost of calling the function is $E L C A(v, C H)$ once is $O(|C H| . k d \log |S|)$ or $\mid$ child_elcacan $(v)|k d \log | S \mid$ (see Figure 2). The accumulated total cost of calling isELCA is $O\left(\sum_{v \in e l c a \_c a n\left(S_{1} ; S_{2}, \ldots, S_{k}\right)} \mid\right.$ child_elcacan $\left.(v)|k d \log | S \mid\right)$. Let

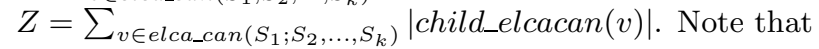
$\left|e l c a \_c a n\left(S_{1} ; S_{2}, \ldots, S_{k}\right)\right| \leq\left|S_{1}\right|$ and $\mid$ child_elcacan $(v) \mid \leq$ $\left|S_{1}\right|$. Each node in elca_can $\left(S_{1} ; S_{2}, \ldots, S_{k}\right)$ increases the value of $Z$ by at most one (see Figure 4 ). Thus $O\left(\sum_{v \in e l c a \_c a n}\left(S_{1} ; S_{2}, \ldots, S_{k}\right) \mid\right.$ child_elcacan $\left.(v) \mid\right)=O\left(\left|S_{1}\right|\right)$. Therefore the time complexity of the Indexed Stack algorithm is $O\left(\left|S_{1}\right| k d \log |S|\right)$.

The number of disk access needed by the Indexed Stack algorithm is $O\left(k\left|S_{1}\right|\right)$ because for each node in $S_{1}$ the Indexed Stack algorithm just needs to find the left and match nodes in each one of the other $k-1$ keyword lists. Note that the number of disk accesses of the Indexed Stack algorithm cannot be more than the total number of blocks of all keyword lists on disk because the algorithm accesses all keyword lists strictly in order and there is no repeated scan on any keyword list. Since B+ tree implementations usually buffer non-leaf nodes in memory, we assume the number of disk accesses of a random search in a keyword search is $O(1)$ as in $[10,22]$. The complexity analysis of the Indexed Stack, the two algorithms in [10], DIL and RDIL are summarized in Table 1 for both main memory and disk accesses for finding all query answers and only top $m$ query answers where $\left|S_{1}\right|(|S|)$ is the occurrence of the least (most) frequent keyword in the query, $B$ is the total number of blocks of all inverted lists on disk, $d$ is the maximum depth of the tree and $p$ is the maximum number of children of any node in the tree.

\subsubsection{Running Example}

We illustrate the algorithm using the query "XML David" on the data of Figure 1. Figure 7 shows the states of the stack after the processing of each node in $S_{1}$ for computing elca $\left(S_{1} ; S_{2}\right)$. The caption under each figure describes which node $v_{1}$ in $S_{1}$ has just been processed, the id of the node $e l c a \_c a n_{v_{1}}=e l c a \_c a n\left(v_{1}\right)$, which of the four cases in Figure 5 has happened, and the pop/push actions that happened.

Figures $7(\mathrm{a}), 7(\mathrm{~b})$, and $7(\mathrm{c})$ show the processing of the first three $S_{1}$ nodes, 0.1.1, 0.2.1.1 and 0.2.2.1.1. The case of Figure 5(b) is applied.

Figure $7(\mathrm{~d})$ shows the processing of the node 0.3.2.1.1. The case of Figure 5(c) is applied. The two nodes 0.2 .2 and 0.2 are popped out from the stack and determined to be $E L C A$ nodes; the $C H$ field associated with the node 0 is updated with the addition of the node 0.2 ; and elca_can (0.3.2.1.1) $=0.3 .2$ is pushed onto the stack.

Figure $7(\mathrm{e})$ shows the result of processing 0.3.3.1.1 from $S_{1}$. Note that elca_can $v_{v_{1}}=0.3 .3$. The processing for the case of Figure 5(c) is applied. The node 0.3.2 is popped out and reported as an ELCA. Also 0.3 .2 is stored in the $S I B$ field of the entry associated with 0.3.3. Figure $7(\mathrm{f})$ shows the processing of the node 0.3.4.1.1 from $S_{1}$ which is similar to the processing shown in Figure $7(\mathrm{e})$. The node 0.3 .3 is popped out and reported as an ELCA, and added to the SIB field of the stack entry associated with 0.3.4. Note that the $E L C A \_C A N$ node 0.3 has not been seen yet.

The processing for the node 0.3 shown in Figure $7(\mathrm{~g})$ is interesting in that it picks up the nodes previously stored in $S I B$ and uses it to update the $C H$ field of the stack 


\begin{tabular}{|l|l|l|}
\hline & number of disk accesses & main memory complexity \\
\hline IS & $O\left(k\left|S_{1}\right|\right)$ & $O\left(k d\left|S_{1}\right| \log |S|\right)$ \\
\hline DIL & $O(B)$ & $O(k d|S|)$ \\
\hline RDIL & $O\left(k^{2} d|S| p \log |S|+k^{2} d|S|^{2}\right)$ & $O\left(k^{2} d|S| p \log |S|+k^{2} d|S|^{2}\right)$ \\
\hline
\end{tabular}

Table 1: Main memory and Disk Complexity Analysis of Indexed Stack, DIL and RDIL

entry associated with 0.3 . Without this action, we cannot determine whether the node 0.3 is an $E L C A$ or not because some of its child ELCA_CAN nodes (0.3.2, 0.3.3 and 0.3.4) have been seen and they have to been stored. The node 0.3.4 is popped out and determined to be an ELCA node.

Figure 7(h) shows the processing of the last node 0.4.2.1.1 from $S_{1}$ which is similar to the processing shown in Figure $7(\mathrm{~d})$. The node 0.3 is popped out and determined to be an $E L C A$ node. The node 0.4 .2 is pushed onto the stack. At this stage every node in $S_{1}$ has been processed. Figure 7(i) shows that after cleaning up the stack, the stack becomes empty and nodes 0.4 .2 and 0 are determined to be $E L C A$ nodes.

\subsubsection{Algorithm Optimization}

To emphasize the key ideas behind the Indexed Stack algorithm and for presentation simplicity, we did not present some optimization techniques in the implementation of the algorithm shown in Figure 6.

Incremental isELCA(). Notice that we can do without storing the child or descendant ELCA_CAN nodes of an $E L C A \_C A N$ node in the stack. That is, we can remove the $C H$ field in the structure of a stack entry. The above can be achieved by the following two changes: i) extending the computation of elca_can $(v)$ along with an array of $E L C A \_C A N$ witness nodes of $e l c a \_c a n(v)$; ii) changing the function is $E L C A$ 's signature accordingly to

is $E L C A(l, W N)$ where $l$ is an $E L C A \_C A N$ node and $W N$ is the list of l's $E L C A \_C A N$ witness nodes. The idea is that some of the $E L C A \_C A N$ witness nodes of elca_can $(v)$ kept along the way of computing elca_can $(v)$ may be $E L C A$ witness node for $e l c a \_c a n(v)$. If an ELCA_CAN witness node $x$ is also an $E L C A$ witness node for elca_can $(v)$ in a set $S_{i}$, then there is no need in $i s E L C A()$ to search for $E L C A$ witness nodes for elca_can $(v)$ in $S_{i}$. For example in the stack state shown in Figure 7(h), the child $E L C A \_C A N$ node 0.2 of the node 0 is stored in the $C H$ field associated with the node 0 at the bottom of the stack. Instead of carrying the child ELCA_CAN 0.2 of the node 0 from the state shown in Figure $7(\mathrm{~d})$ to the state shown in Figure $7(\mathrm{~h})$, we can at the step shown in Figure $7(\mathrm{~d})$ update the witness node of 0 from $[0.1 .1,0.2 .2 .2 .1]$ to $[0.1 .1,0.3 .1 .1]$ after 0.2 .2 and 0.2 are popped out and before 0.3 is pushed onto the stack, and update at the step shown in Figure $7(\mathrm{e})$ the witness node array of 0 from $[0.1 .1,0.3 .1 .1]$ to $[0.1 .1,0.4 .1 .1]$. In the last step (Figure $7(\mathrm{i})$ ) after popping out 0.4 .2 , we update the witness node array of 0 to $[0.1 .1,0.5 .1]$ and determine that 0 is an $E L C A$ node. Essentially, we remove the need of storing child ELCA_CAN nodes in the stack's CH fields and carrying them around by reusing the computation of elca_can() in the function isELCA() and by doing some of the work in $i s E L C A()$ (searching for $E L C A$ witness nodes) as early as possible.

Reducing $|S I B|$. Assume at some point in the processing of the algorithm, the following list of $E L C A \_C A N$ nodes are

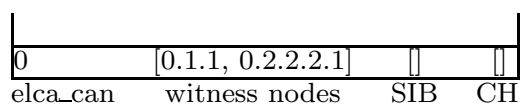

(a) $v_{1}=0.1 .1 ;$ elca_can $v_{1}=0$; Figure $5(\mathrm{~b})$; push 0 to stack.

\begin{tabular}{|lccc|}
\hline & & & \\
\hline 0.2 & {$[0.2 .1 .1,0.2 .2 .2 .1]$} & {[} & {[]} \\
\hline 0 & {$[0.1 .1,0.2 .2 .2 .1]$} & {[]} & ] \\
\hline elca_can & witness nodes & SIB & CH
\end{tabular}

(b) $v_{1}=0.2 .1 .1$ : elca_can $v_{1}=0.2$; Figure $5(\mathrm{~b})$; push 0.2 to stack.

\begin{tabular}{|lccr||}
\hline & & & \\
\hline 0.2 .2 & {$[0.2 .2 .1 .1,0.2 .2 .2 .1]$} & ] & \\
\hline 0.2 & {$[0.2 .1 .1,0.2 .2 .2 .1]$} & ] & ] \\
\hline 0 & {$[0.1 .1,0.2 .2 .2 .1]$} & ] & ] \\
\hline elca_can & witness nodes & SIB & CH
\end{tabular}

(c) $v_{1}=0.2 .2 .1 .1$ : elca_can $v_{v_{1}}=0.2 .2$; Figure $5(\mathrm{~b})$; push 0.2 .2 to stack.

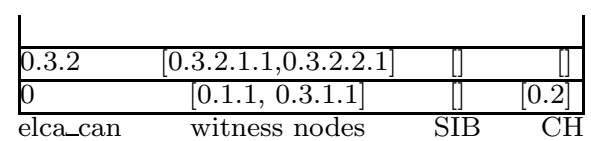

(d) $v_{1}=0.3 .2 .1 .1:$ elca_can $v_{1}=0.3 .2 ;$ Figure $5(\mathrm{c})$; pop out 0.2 .2 and 0.2 and determine them as ELCAs; add 0.2 to top entry's $\mathrm{CH}$; push 0.3.2 to stack.

\begin{tabular}{|lccr||}
\hline & & & \\
\hline 0.3 .3 & {$[0.3 .3 .1 .1,0.3 .3 .2 .1]$} & {$[0.3 .2]$} & \\
\hline 0 & {$[0.1 .1,0.4 .1 .1]$} & {[]} & $0.2,0.3 .2]$ \\
\hline elca_can & witness nodes & SIB & CH
\end{tabular}

(e) $v_{1}=0.3 .3 .1 .1$ : elca_can $v_{1}=0.3 .3$; Figure 5(c); pop out 0.3 .2 and determine it as an ELCA; add

0.3.2 to 0.3.3's SIB; push 0.3.3 to stack.

\begin{tabular}{|lcccr|}
\hline \multicolumn{4}{|c|}{} \\
\hline 0.3 .4 & {$[0.3 .4 .1 .1,0.3 .4 .2 .1]$} & {$[0.3 .2,0.3 .3]$} & ] \\
\hline 0 & {$[0.1 .1,0.4 .1 .1]$} & {[]} & $0.2,0.3 .2,0.3 .3]$ \\
\hline elca_can & witness nodes & SIB & CH
\end{tabular}

(f) $v_{1}=0.3 .4 .1 .1 ;$ elca_can $v_{1}=0.3 .4$; Figure $5(\mathrm{c})$; pop out 0.3.3 and determine it as an ELCA; add 0.3.3 to 0.3.4's SIB; push 0.3.4 to stack.

\begin{tabular}{|lrr||r|}
\hline \multicolumn{4}{|c|}{} \\
\hline 0.3 & {$[0.3 .1 .1,0.3 .4 .2 .1]$} & ] & {$[0.3 .2,0.3 .3,0.3 .4]$} \\
\hline 0 & {$[0.1 .1,0.4 .1 .1]$} & ] & {$[0.2,0.3 .2,0.3 .3,0.3 .4]$} \\
\hline elca_can & witness nodes & SIB & CH
\end{tabular}

(g) $v_{1}=0.3 .5 .1 ;$ elca_can $v_{1}=0.3$; Figure $5(\mathrm{~d})$; pop out 0.3.4 and determine it as an ELCA; add 0.3.4 entry's SIB list and 0.3 .4 to 0.3 's $\mathrm{CH}$; push 0.3 to stack.

\begin{tabular}{|lcc|l|}
\hline 0.4 .2 & {$[0.4 .2 .1 .1,0.4 .2 .2 .1]$} & ] & \\
\hline 0 & {$[0.1 .1,0.4 .1 .1]$} & ] & {$[0.2,0.3 .2,0.3 .3,0.3 .4,0.3]$} \\
\hline elca_can & witness nodes & SIB & CH
\end{tabular}

(h) $v_{1}=0.4 .2 .1 .1 ;$ elca_can $v_{1}=0.4 .2$; Figure $5(\mathrm{c})$; pop out 0.3 and determine it as an ELCA; push 0.4.2 to stack.

(i) No more "XML" nodes: clean up the stack; pop out 0.4.2 and 0 and determine them as ELCAs; Stack becomes empty.

\section{Figure 7: States of stack during evaluation of "XML} David" 


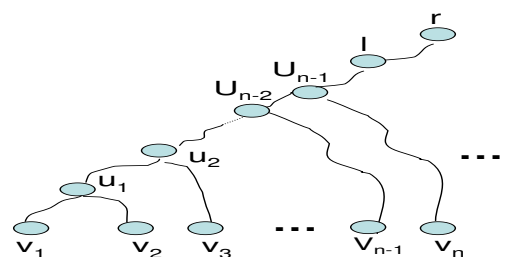

Figure 8: Optimizing the history information of an $E L C A \_C A N$ node

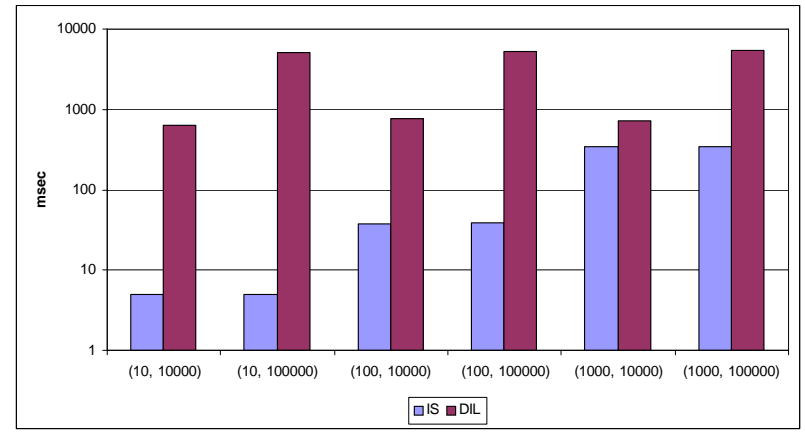

Figure 9: Finding all query answers (evaluating the Indexed Stack algorithm and DIL): queries contain two keywords; frequencies shown on $\mathrm{X}$-axis

computed in the exact order as they appear $-r, v_{1}, v_{2}, \ldots$, $v_{n}, l$ (See Figure 8). The algorithm presented in Figure 6 will at some point push the node $r$ onto the stack; push $v_{1}$ onto the stack; pop out $v_{1}$, push $v_{2}$, and add $v_{1}$ to the $S I B$ field associated with $v_{2}$; pop out $v_{2}$, push $v_{3}$, and add $v_{1}$ and $v_{2}$ to the $S I B$ field associated with $v_{3}$. When the algorithm pushes $v_{n}$ onto the stack, the $S I B$ field associated with $v_{n}$ contains $v_{1}, \ldots, v_{n-1}$. We only describe the basic idea of the optimization to reduce the number of nodes stored in the $S I B$ field. The idea is that we only need to store $v_{1}$ in the $S I B$ field of $v_{2} ; u_{1}$ in the $S I B$ field of $v_{3} ; \ldots ; u_{n-2}$ in the $S I B$ field of $v_{n}$.

\section{EXPERIMENTAL EVALUATION}

System Implementation and Setup We have implemented in Java a prototype called XKeywordSearch to evaluate the proposed Indexed Stack algorithm and the two core algorithms in [10].

We have run XKeywordSearch on both real and synthetic data, respectively, DBLP [5] and XMark [17] data. The experiments have been done on a $766 \mathrm{MHz}$ computer with $512 \mathrm{MB}$ of RAM. We only report the experimental results on the DBLP data in this paper; the results on XMark are similar.

The DBLP data was first grouped by journal and conference names, then by years. The size of the XML file of DBLP data after grouping is $120 \mathrm{MB}$. The depth of the DBLP tree is 10 ; the number of distinct keywords is 180,126 ; the number of nodes in the tree is $6,267,592$.

We evaluated the Indexed Stack algorithm, DIL and RDIL discussed in Section 1 for the ELCA query semantics by varying the number and frequency of keywords both on hot cache and on cold cache. We report only results on hot cache in this paper. The relationships among three evalu- ated algorithms on cold cache are similar in the sense that if one algorithm wins another algorithm in the hot cache it also wins in the corresponding cold cache experiment but the differences are smaller because of dominance of the disk access. For example, in the hot cache experiments shown in Figure 9, the response time of the Indexed Stack algorithm for a query with two keywords of frequencies of 10 and 10000 is below 10 milliseconds; in the cold cache experiments, the response time of the Indexed Stack algorithm for the same query is close to 100 milliseconds. But the response time of the DIL algorithm does not increase significantly from hot cache to cold cache experiments.

One hundred queries were randomly selected for each experiment by a script. Note that when the script fails to choose a sufficient number of keywords of a specified frequency, it chooses keywords with frequencies close to the specified frequency. Each query was run three times and the average time was reported.

Search Performance First, we compare the search performances of the Indexed Stack (IS) algorithm and the DIL algorithm for finding all query results. There is no point to run the RDIL algorithm to find all query results because it is designed for returning top $m$ answers and it has higher complexity than the IS algorithm. For space reason, we do not report experiments where the response time of both algorithms are less than 100 milliseconds.

In Figure 9 each query contains two keywords. The performance of the DIL algorithm degrades linearly when the size of the large inverted list increases, while the response time of the IS algorithm is almost constant, linear in the size of the smaller keyword list, and its performance is orders of magnitude better than DIL.

In the experiments shown in Figure 10, we vary the number of keywords from two to five. Each query has a keyword of small frequency shown on the top of Figure 10, while the frequency of all other keywords in the query is fixed at 100000 . We vary the small frequency from 10 to 10000 . As can been seen from Figure 10, when the number of the keywords is fixed, the performance of the DIL algorithm is essentially independent of $\left|S_{1}\right|$ when the small frequency increases from 10 to 10000, while the performance of the IS algorithm degrades linearly when the size of the small frequency increases. When the small frequency is fixed, the performance of IS is essentially constant while the performance of DIL degrades linearly when the number of keywords increases. As demonstrated in Figure 9, Figure 10 shows that the performance of the IS algorithm is orders of magnitude better than DIL.

We also stress tested the Indexed Stack algorithm on queries where all keywords have the same frequency. The experiments showed that although DIL often performs better than IS, the difference is not significantly. It is less than $5 \%$ in most experiments and less than $12 \%$ on average.

Next, we compare the search performance of the Indexed Stack algorithm and the RDIL algorithm for returning only the top ten query results. The DIL algorithm is not evaluated in this set of experiments because both the DIL and IS algorithms have to find all query results to determine the top ten answers and the experiments shown in Figures 9 and 10 in finding all query results have showed that IS is a better choice than DIL. As discussed in Section 3, there is no guarantee that RDIL can always find the top ten queries without having to compute all query results. 


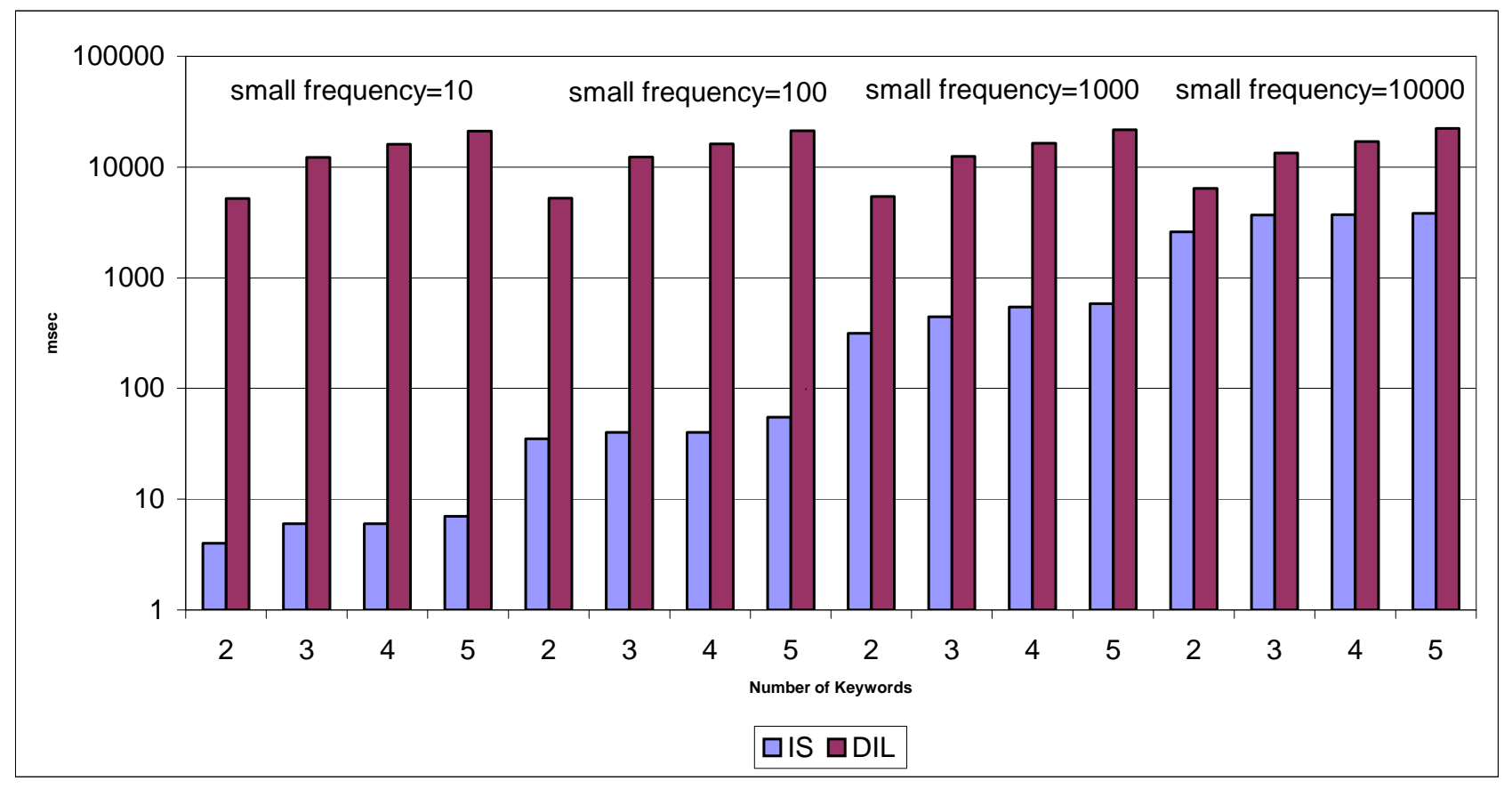

Figure 10: Finding all query answers (evaluating the Indexed Stack algorithm and DIL): varying the number of keywords from 2 to 5 ; large Frequency $=100000$

We evaluated the queries in Figure 9 and Figure 10 and reported the time on returning the top ten query answers in Figure 11 and Figure 12 respectively. We used a ranking module that is identical to the one used in the experiments of [10]. Both Figure 11 and Figure 12 show that the Indexed Stack algorithm performed significantly better than the RDIL algorithm.

There is a space where RDIL can outperform IS (and DIL) and here is a scenario that exhibits the conditions under which this happens. Consider a query " $w_{1} w_{2}$ " on a XML document that contains a large number of occurrences of $w_{1}$ and $w_{2}$, and only ten pairs of $w_{1}$ and $w_{2}$ have non-root nodes as their lowest common ancestors. Assume that the ten pairs of $w_{1}$ and $w_{2}$ nodes have higher ranking than all other $w_{1}$ and $w_{2}$ nodes before them in the document. The RDIL algorithm outperforms the IS algorithm for the above query " $w_{1}$ $w_{2}$ " because the IS algorithm has to scan to the end of one of the two inverted lists to return the top ten answers while the RDIL algorithm starts from inverted lists sorted by ranking scores and can terminate much earlier than IS. As one direction of future work, we plan to investigate how to return top $m$ answers without having to completely scan the smallest inverted list, by either adjusting the ranking mechanism or relaxing the exact top $m$ requirement to approximate top $m$ query answers.

\section{CONCLUSIONS}

We have presented an efficient keyword search algorithm, named Indexed Stack, that returns nodes that contain all instances of all keywords in the query, after excluding the keyword instances that appear under nodes whose children already contain all keyword instances based on the query semantics proposed in [10]. We demonstrated the superiority of the Indexed Stack algorithm over DIL and RDIL in

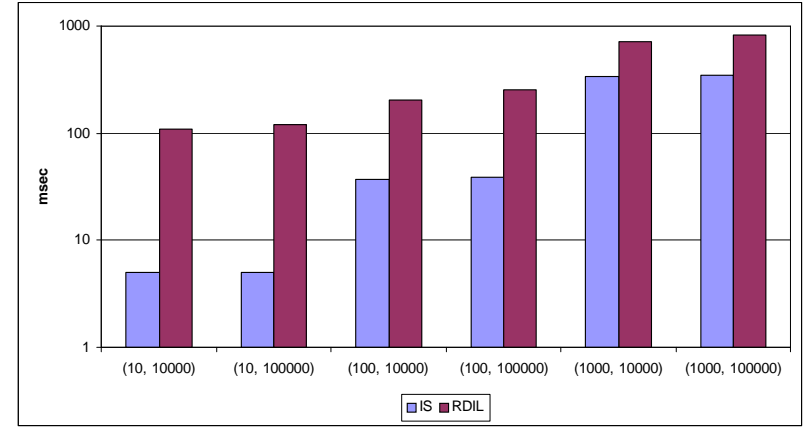

Figure 11: Finding top 10 query answers (evaluating the Indexed Stack algorithm and RDIL): queries contain two keywords; frequencies shown on $\mathrm{X}$-axis

[10] both analytically and experimentally. We showed that the complexity is $O\left(k d\left|S_{1}\right| \log |S|\right)$ where $k$ is the number of keywords in the query, $d$ is the depth of the tree and $\left|S_{1}\right|$ $(|S|)$ is the occurrence of the least (most) frequent keyword in the query. In comparison, the complexity of the best prior work algorithm is $O(k d|S|)$.

\section{REFERENCES}

[1] S. Agrawal, S. Chaudhuri, and G. Das. DBXplorer: A system for keyword-based search over relational databases. In ICDE, 2002.

[2] S. Amer-Yahia, C. Botev, and J. Shanmugasundaram. TeXQuery: A full-text search extension to XQuery. In $W W W, 2004$.

[3] G. Bhalotia, A. Hulgeri, C. Nakhe, S. Chakrabarti, and S. Sudarshan. Keyword searching and browsing in databases using BANKS. In ICDE, 2002. 


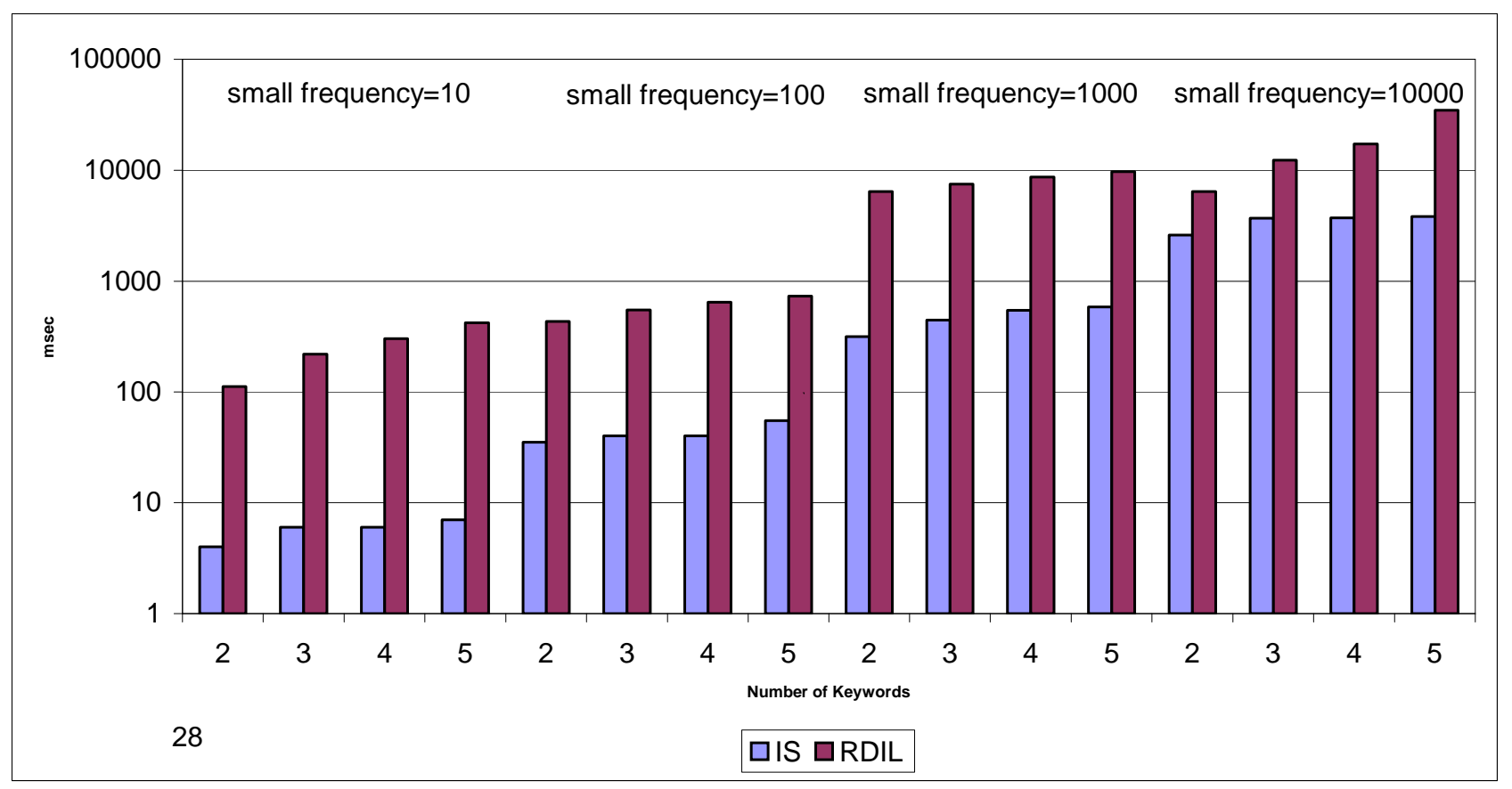

Figure 12: Finding top 10 query answers (evaluating the Indexed Stack algorithm and RDIL): varying the number of keywords; large Frequency $=100000$

[4] S. Cohen, J. Namou, Y. Kanza, and Y. Sagiv. XSEarch: A semantic search engine for XML. In $V L D B, 2003$.

[5] DBLP. http://www.informatik.uni-trier.de/ ley/db.

[6] D. Florescu, D. Kossmann, and I. Manolescu. Integrating keyword search into XML query processing. In $W W W 9,2000$.

[7] N. Fuhr and K. GroSSjohann. XIRQL: A Query Language for Information Retrieval in XML documents. In SIGIR, 2001.

[8] H. Garcia-Molina, J. Ullman, and J. Widom. Database System Implementation. Prentice-Hall, 2000.

[9] R. Goldman, N. Shivakumar, S. Venkatasubramanian, and H. Garcia-Molina. Proximity Search in Databases. In $V L D B, 1998$.

[10] L. Guo, F. Shao, C. Botev, and J. Shanmugasundaram. XRANK: Ranked keyword search over XML documents. In SIGMOD, 2003.

[11] V. Hristidis and Y. Papakonstantinou. Discover: Keyword search in relational databases. In $V L D B$, 2002.

[12] V. Hristidis, Y. Papakonstantinou, and A. Balmin. Keyword proximity search on XML graphs. In $I C D E$, 2003.

[13] V. Kacholia, S. Pandit, S. Chakrabarti, S. Sudarshan, R. Desai, and H. Karambelkar. Bidirectional expansion for keyword search on graph databases. In $V L D B, 2005$.

[14] Y. Li, C. Yu, and H. V. Jagadish. Schema-free XQuery. In $V L D B, 2004$.

[15] Z. Liu and Y. Chen. Identifying meaningful return information for xml keyword search. In SIGMOD, 2007.
[16] D. Quass, A. Rajaraman, Y. Sagiv, J. D. Ullman, and J. Widom. Querying semistructured heterogeneous information. In Deductive and Object-Oriented Databases, pages 319-344, 1995.

[17] R.Busse et al. XMark, the XML benchmark project, http://monetdb.cwi.nl/xml.

[18] A. Schmidt, M. L. Kersten, and M. Windhouwer. Querying XML documents made easy: Nearest concept queries. In ICDE, 2001.

[19] C. Sun, C. Y. Chan, and A. K. Goenka. Multiway slca-based keyword search in xml data. In $W W W$.

[20] A. Theobald and G. Weikum. Adding relevance to XML. In WebDB, 2000.

[21] A. Theobald and G. Weikum. The index-based XXL search engine for querying XML data with relevance ranking. In EDBT, 2002.

[22] Y. Xu and Y. Papakonstantinou. Efficient keyword search for smallest LCAs in XML databases. In SIGMOD, 2005. 